\title{
Las Redes Sociales y su RePercusión en el Lenguaje de la Población UNIVERSITARIA
}

\author{
Social Networks and its Impact on the Language of the University Population
}

\author{
Claudia Desiré Díaz CJahua ${ }^{1}$
}

\begin{abstract}
Resumen
La tecnología y la globalización son fenómenos que han generado transformaciones en la forma de comunicarnos e interactuar con nuestro entorno, y la educación no es ajena a esta nueva realidad. Dentro de este contexto, las redes sociales en Internet han adquirido una gran relevancia debido a que existe también un uso generalizado de los dispositivos móviles. Cada vez es más difícil que los jóvenes puedan contar con la capacidad de discriminar su interacción en los entornos online y offline, lo cual, quiérase o no, repercute en el uso del lenguaje, creando nuevas formas de comunicación. En el presente artículo, se describe la incidencia del uso de las redes sociales en el proceso de enseñanza - aprendizaje, así como en el ejercicio de las habilidades lingüísticas de los estudiantes universitarios.
\end{abstract}

Palabras claves: Internet, redes sociales, lenguaje verbal, lenguaje no verbal, competencias comunicación, habilidades, lingüística, enseñanza, aprendizaje.

\begin{abstract}
Technology and globalization are phenomena that have generated transformations in the way we communicate and interact with our environment, and education is not stranger to this new reality. Within this context, social networks on the Internet have acquired great relevance because there is also a generalized use of mobile devices. It is increasingly difficult for young people to have the ability to discriminate their interaction in online and offline environments, which, whether they like it or not, has an impact on the use of language, creating new forms of communication. In this article, we describe the incidence of social networks in the teaching - learning process, as well as the exercise of the linguistic abilities of university students.
\end{abstract}

Key words: Internet, social networks, verbal, non-verbal, communication skills, abilities, Linguistics, teaching and learning.
$\mathfrak{E}$ siglo XXI tiene como una de sus características principales la inclusión de la tecnología y la globalización, lo cual ha provocado notables cambios en la sociedad. Las actividades socioeconómicas y culturales del ser humano tienden a accionar o vincularse con la tecnología, representada principalmente por la Internet y las redes sociales. Actualmente, la Internet se ha establecido como el medio tecnológico ideal para acceder a distinto tipo de información y posibilitar la comunicación de manera dinámica e instantánea. Es más, estos beneficios se han masificado gracias al uso de los teléfonos inteligentes, donde las redes sociales tienen una importancia preponderante.

El desarrollo de estos medios de información ha traído consigo diferentes plataformas, tales como el Messenger, hasta la aparición de las redes sociales horizontales como el Facebook y Twitter. Pero, pese a que las redes sociales han simplificado la vida, estas han perjudicado enormemente el progreso y desempeño de las capacidades lingüísticas de las personas,

1 Docente del Departamento de Humanidades de la Universidad Privada del Norte. 
alterando el lenguaje verbal y no verbal, en especial, en los jóvenes y adolescentes, muchos de ellos estudiantes.

En el uso de las redes sociales se puede visualizar el mal empleo de las reglas gramaticales, graves errores de ortografía, la falta de precisión léxica, la incoherencia de los textos, el reemplazo de oraciones por dibujos o símbolos, entre otros aspectos. Esto afecta de manera significativa el desarrollo lingüístico de los jóvenes y adolescentes, base crucial para el ejercicio de su vida académica y profesional.

\section{ORIGEN Y EVOLUCIÓN DE LAS REDES SOCIALES}

La aparición de las redes sociales se remonta al año 1995 con la creación del sitio web "Classmates.com", el cual permite que las personas puedan retomar el contacto con personas de su pasado. Esta red fue creada por Randy Conrads y fue de gran alcance, principalmente, en EE.UU. y Canadá. Posteriormente, aparece "SixDegrees", website creada en 1997. Esta página llegó a tener una cobertura de tres millones de usuarios. Luego, en el año 2002, aparecieron sitios web ofreciendo las redes de círculos de amigos en línea.

Las redes sociales propiamente dichas se originaron a raíz de la crisis financiera e informática que muchas empresas sufrieron en el 2003, lo cual derivó en el cierre de sus páginas web debido a la escasez de visitas. Es en aquel entonces cuando los usuarios cobran protagonismo y tres norteamericanos, basándose en la mensajería instantánea y los foros de discusión, crean en ese momento sendas empresas destinadas a que los internautas puedan hablar entre ellos $\mathrm{y}$ conocerse mejor. ${ }^{(1)}$
En los años posteriores, con la llegada de sitios tales como Friendster, MySpace, Ecademy, Soflow, entre otros, existían más de 200 sitios de redes sociales. Finalmente, en el 2004 se crea Facebook, cuyo alcance, en un principio, eran solo redes universitarias. En esta red los usuarios del sitio estaban obligados a proporcionar las direcciones de correo electrónico asociadas con las instituciones educativas. Más adelante, la red se amplió para incluir a todos los usuarios potenciales de Internet. Desde entonces, diversas redes se han creado, unas permanecen y otras ya han desaparecido. Un poco antes del 2009 hasta la actualidad, los principales competidores a nivel mundial son: Facebook, Twitter, Google+ e Instagram. ${ }^{(2)}$

\section{Características de las Redes Sociales}

Las redes sociales se caracterizan por ser un área de participación ciudadana, un medio de comunicación cada vez más utilizado en detrimento del correo electrónico, una comunidad o punto de encuentro en el que los usuarios construyen, comparten y desarrollan conocimientos. Además, es un espacio que permite suscripciones a eventos, noticias y boletines, también una zona personal para acceder a servicios personalizados, presenta alertas, es de fácil acceso a las mismas a través del ordenador, móviles, tablet, etc., y tiene un papel facilitador para el logro de resultados. ${ }^{(6)}$

En diversos espacios de la red podemos encontrar un conjunto de rasgos que caracterizan a las redes sociales:

- Tienen como base al usuario, es decir, son construidas y dirigidas por los mismos usuarios, quienes además incrementan su capacidad con el contenido vertido en ellas.

- Poseen un conjunto de salas de chat y foros, lo cual las hace interactivas. En otras 
palabras, contienen una serie de aplicaciones basadas en una red de juegos, como una forma de conectarse y entretenerse con las amistades.

- Permiten establecer relaciones y descubrir nuevos contactos con quienes se comparte intereses comunes. Asimismo, admiten la posibilidad de volver a conectar con amistades con las que se ha perdido contacto desde hace ya tiempo atrás.

- Posibilitan el intercambio de información e intereses, mediante las publicaciones y comentarios que difunden los usuarios a través de su red de contactos y subcontactos.

- Ofrece una variedad de servicios, tales como el intercambio de información, fotografías, servicios de telefonía, juegos, chat, foros.

\section{ReDes SOCIALES EN EL PROCESO DE ENSEÑANZA- APRENDIZAJE}

La educación ha sido una de las disciplinas más beneficiadas con la irrupción de las nuevas tecnologías, especialmente las relacionadas a la Web 2.0. Por ello, resulta fundamental conocer y aprovechar la batería de nuevos dispositivos digitales, que abren inexploradas potencialidades a la educación y la investigación. Incluso en el argot académico algunos ya hablan del "Aprendizaje 2.0". (3)

Esto se da debido a que las redes sociales hacen más factible el trabajo en equipos y la acción colaborativa. Asimismo, es un espacio idóneo para que los estudiantes empleen estas redes como herramientas motivadoras que les faciliten la realización de tareas y les sirvan de apoyo en su proceso de aprendizaje.

Muchos docentes buscan aprovechar el tiempo que invierten los estudiantes en su uso, para dejar tareas que permitan complementar lo aprendido en clases. El primer uso de las redes sociales en el ámbito educativo aparece con el empleo de herramientas de comunicación interpersonal y colaboración en red, en este sentido, son numerosas las posibilidades que brindan las redes sociales en la práctica docente. ${ }^{(4)}$

Estas particularidades brindan la posibilidad de potenciar diversos aspectos como la participación del alumnado en el proceso de enseñanza y aprendizaje, aprendizaje autónomo, interacción y motivación de los estudiantes, creatividad del estudiante y la creación de redes de colaboración e intercambio con continuidad. ${ }^{(5)}$

Es la misma actividad educativa la que demanda el uso de estos nuevos recursos y materiales tecnológicos puesto que los estudiantes se encuentran en constante interacción, y las redes les resultan bastante útiles para la organización coordinación y planificación de sus actividades académicas.

\section{REDES SOCIALES VERTICALES}

Son aquellas que están concebidas sobre la base de un eje temático agregador y cuyo objetivo es el de congregar en torno a una temática definida a un colectivo concreto. En función de su especialización, pueden clasificarse a su vez en:

a. Redes sociales verticales profesionales: Generan relaciones profesionales entre los usuarios. Los ejemplos más representativos son Viadeo, Xing y Linked In.

b. Redes sociales verticales de ocio: Congrega a colectivos que desarrollan actividades de ocio, deporte, usuarios de videojuegos, fans, etc. Los ejemplos más representativos son Wipley, MinubeDogster, Last.FM y Moterus. 
c. Redes sociales verticales mixtas: Ofrecen a usuarios y empresas un entorno específico para desarrollar actividades tanto profesionales como personales en torno a sus perfiles: Yuglo, Unience y PideCita. ${ }^{(7)}$

\section{REDES SOCIALES HORIZONTALES}

Son aquellas dirigidas a todo tipo de usuario y sin una temática definida. Se basan en una estructura de celdillas permitiendo la entrada y participación libre y genérica sin un fin definido, distinto del de generar masa. Los ejemplos más representativos del sector son Facebook, Orkut, Identica, Twitter. ${ }^{(8)}$

Estas redes se caracterizan por ofrecer gran variedad de aplicaciones y/o funcionalidades que permiten a los usuarios prescindir de herramientas de comunicación externas. Se les llama también generalistas, puesto que no están enfocadas a alojar un tema concreto sino que en ellas cabe cualquier cosa: trabajo, ocio, amigos, familia, deportes, literatura, etc., de todo puede hablarse y las relaciones pueden ser de lo más heterogéneas. ${ }^{(9)}$

\section{Uso Del LeNGUAJE}

El lenguaje ha sido definido y estudiado desde diferentes puntos de vista. Desde luego, se puede afirmar que el lenguaje ha favorecido la transformación de la conciencia humana, ya que trajo como resultado nuevas formas de pensamiento. Se puede definir al lenguaje como la facultad innata del ser humano para expresar sus pensamientos, muchas veces, mediante una lengua. Es un instrumento de comunicación que implica un conjunto de signos y reglas producidas por el hombre y aceptados por determinada comunidad.

Se puede inferir que las interpretaciones que se hacen del término lenguaje están unidas, en la mayor parte de los casos, a los intereses particulares de cada teórico que propone la definición. (10) Por un lado, Chomsky (1957) expone que el lenguaje es un conjunto finito o infinito de oraciones, cada una de ellas de longitud finita y construida a partir de un conjunto finito de elementos. Por otro lado, Luria (1977) sostiene que lenguaje es un sistema de códigos con cuya ayuda se designan los objetos del mundo exterior, sus acciones, cualidades y relaciones entre los mismos.

Lo que tienen en común estas definiciones es sostener que el lenguaje es un sistema de signos que permite a los seres humanos expresarse y llegar a tender puentes de comunicación entre ellos.

\section{Características del Lenguaje}

- Los signos que componen el lenguaje puede dividirse en dos partes: el significado, que es la imagen mental del concepto que se está representando, y el significante, que es la forma material del signo, ya sea una palabra o una imagen.

- El lenguaje es al mismo tiempo innato y aprendido, ya que las personas nacen con una capacidad lingüística hereditaria, que consiste en una gramática universal común a todos los seres humanos, la cual se irá transformando en una gramática particular (con una estructura propia) a medida que la persona se desarrolle en un contexto social y familiar.

- El lenguaje está permanentemente en un proceso de cambio y evolución, que se produce por transformaciones acumulativas en el habla cotidiana de las personas, proceso que se conoce con el nombre de cambio lingüístico, y que puede dar origen a lenguas muertas (idiomas que dejan de 
transformarse y utilizarse, como el latín) y lenguas vivas (como el castellano, el inglés, el francés, el mandarín, etc.).

- El lenguaje es en sí mismo arbitrario, ya que la asociación entre ciertos símbolos/sonidos por un lado, y ciertos objetos o ideas, no tiene una relación necesaria sino que surge de la práctica y de convenciones sociales que pueden variar entre diferentes grupos o sociedades.

\section{EMPLEO DEL LeNGUAJE}

\section{EN LOS ENTORNOS VIRTUALES}

Es en los códigos verbales escritos donde en estos últimos tiempos se han visto grandes y alarmante cambios, a manos de un grupo social conformado por adolescentes, que en su afán de comunicarse a la brevedad y masivamente han modificado la escritura de diversos vocablos a satisfacción propia, ya sea con la intención de agilizar el proceso comunicativo, personalizar sus mensajes o marcar un límite "territorial" ante un grupo. Esto se ve con más claridad en los diálogos que los adolescentes mantienen en las redes sociales o aplicaciones de mensajería instantánea como WhatsApp y Facebook Messenger, donde hacen un uso personal y particular del lenguaje, desbaratando cada una de las normas ortográficas ya establecidas. ${ }^{(11)}$

Como sociedad podemos observar que se está gestando un cambio cualitativo en las condiciones lingüísticas y semiológicas de las especie humana. Esto debido a que se está haciendo uso de nuevos recursos, códigos y canales de transmisión de la información. Y en este contexto, son los adolescentes quienes, por las características propias de su edad, son los más propensos a incorporar a sus relaciones cotidianas estos cambios en la forma de comunicarse.
Una nueva forma de expresar sentimientos, emociones o estados de ánimo son los emoticones o emojis. Estos, por ejemplo, complementan un diálogo, permitiendo ubicarnos en un contexto determinado. Vale destacarse que la denominación de emoticón no es casual sino que surgió de la unión de los dos conceptos que representan estas particulares caritas informáticas: emoción e icono. ${ }^{(12)}$ where it is the only mechanism for extruding the $\mathrm{Ca}(2+$

\section{Características del lenguaje EN EL ENTORNO VIRTUAL}

Entre las características del lenguaje que se emplea en el medio virtual, se han podido identificar características de tipo gramatical, léxico y morfológico.

a) Características gramaticales: Implica las diferencias que existen entre los enunciados que se enmarcan en la gramática normativa con las que se dan en el entorno virtual, conocido también como "lenguaje chat". Es decir, se establecen las diferencias que han surgido en el lenguaje chat y se determina hasta qué punto se aparta de los estándares de la gramática normativa.

b) Características léxicas: Hacen referencia a la influencia que el castellano ha recibido de otras lenguas en su desarrollo histórico, siendo una de las más influyentes, la lengua inglesa. Esta influencia se debe entre otros factores al desarrollo tecnológico y globalización del mundo.

c) Características morfológicas: Esto implica el tratamiento que se le da a los sufijos flexivos de número, género y persona. Asimismo, consiste en determinar si existe concordancia entre el sujeto y el predicado (oracional), como también si se presenta entre sustantivos y adjetivos (nominal). ${ }^{(13)}$ 


\section{LOS UNIVERSITARIOS Y LA COMUNICACIÓN ESCRITA}

Las redes sociales ha sido de vital importancia para todos aquellos jóvenes nacidos en la época digital, donde llegan en un simple paso a donde quieran o a comunicarse desde la parte que deseen con la persona que quieren, generando en ellos un lenguaje escrito nuevo, creado y utilizado por ellos.

Por ello, aparecen las nuevas formas de comunicación escrita de los jóvenes por medio de las redes sociales, en las cuales quizá hay mala ortografía, sustitución de letras o sílabas, agregan o suprimen letras a las palabras, utilizando vocablos que pocas personas conocen, además que agregan claves entre ellos, siendo conocido como su código, creando un lenguaje nuevo y moderno. Las redes sociales son espacios de libre expresión, donde han dejado a un lado la normatividad de la lengua española, el lenguaje estándar y abriendo espacio a un lenguaje virtual. ${ }^{(14)}$

\section{LOS MENSAJES EN LAS REDES SOCIALES}

Para que el receptor pueda comprender un mensaje, este tendrá que ser claro y conciso, de modo que se interpreten las ideas tal cual las intentó expresar el emisor. Lamentablemente, dentro de las redes sociales se presenta una comunicación asincrónica. Esto impide que la respuesta sea inmediata y del todo clara, y hasta se podría generar interferencias en la retroalimentación, lo cual desencadenaría en un conflicto en la comunicación.

El lenguaje utilizado en la red social Facebook es prácticamente novedoso y se distingue del empleado en otros medios. Se podría decir que la comunicación mediante esta red social hasta constituye una nueva cultura. La escritura que se utiliza ahí, se convierte en una moda que hace que las personas la trasladen en sus diversos espacios cotidianos, como el colegio, trabajo, entre otros lugares, lo cual es preocupante, pues, en algunos casos, se ha llegado a escribir tal cual uno se expresa oralmente.

La forma de mayor expresión es a través de la escritura, hoy en día se está modificando de una manera muy peculiar, cambiándose radicalmente el lenguaje, donde se modifican palabras o acortándolas para que sean más sencillas de escribir. Muchas personas lo hacen por ser aceptadas en un núcleo de amigos o en la sociedad, por estar a la moda, o por simple pereza, y pues aquellas son las principales causas de este desastre en la ortografía. De esta forma, esto se convierte en un hábito y se expande a muchas más personas, lo cual genera un mundo con degradación del lenguaje absoluto y con muchos errores.

Uno de los cambios en la escritura y lenguaje de los estudiantes al hacer uso de la red social Facebook, es la alternancia de unas letras por otras. Un claro ejemplo es la sustitución de la letra Q por la K, o de la sílaba CA por la K, pues para muchos jóvenes, el hacer uso de estas modificaciones y sustituciones es algo normal y forma parte de una identidad de un grupo o de la moda que se está imponiendo. Las formas de afectar el lenguaje por medio de la red son diversas. Se emplean íconos, abreviaciones, textos cortos, letras supletorias, falta de coherencia en lo que se escribe, degradando la raíz del lenguaje. La flexibilidad de la red, induce a los estudiantes a utilizar un lenguaje a partir de sus gustos e intereses y no un lenguaje que obedece al estándar. ${ }^{(14)}$

Para concluir, se puede afirmar que el uso que se da a las redes sociales en la actualidad tiene un impacto significativo tanto en el ámbito académico, como en el social y personal. Por su parte, el lenguaje también se ha visto afectado, debido a las nuevas formas de expresión propias de este nuevo contexto digital. La 
necesidad de comunicar tanto pensamientos como emociones, ya no se reduce al simple uso de palabras -muchas veces modificadas y transgredidas- sino que hoy recurrimos a elementos visuales dinámicos y estáticos para intentar darnos a entender de manera efectiva. Dada la situación, se hace imperioso reflexionar sobre hasta qué punto se puede admitir la irrupción de estas nuevas formas de interacción sin dejar de velar por el cuidado de nuestro idioma.

\section{RefERENCIAS Bibliográficas}

1. Caldevilla Domínguez D. Las Redes Sociales. Tipología, uso y consumo de las redes 2.0 en la sociedad digital actual. Doc las Ciencias la Inf. 2010;33(1):45-68.

2. GorBrit Social Media. Las Redes Sociales: Origen y evolución [Internet]. 24/06. 2014. URL disponible en: https: / / gorbrit.wordpress.com/2014/06/24/las-redessociales-origen-y-evolucion/

3. Pardo H. Nociones básicas alrededor de la Web 2.0. Planeta 2.0. Inteligencia colectiva o medios fast food. 2007. 27-42 p.

4. Vidal Ledo M, Vialart Vidal MN, Hernández García L. Redes sociales - Social networks. Univ Ciencias Médicas (La Habana - Cuba). 2013;27(1):146-57.

5. Mendiguren T, Meso K, Pérez Dasilva JÁ. El uso de las redes sociales como guía de autoaprendizaje en la Facultad de Comunicación de la UPV/EHU. Rev Didáctica Leng y la Lit. 2012;6:107-22.

6. Hernández M, Posada K, Zavala Y. Influencia del Facebook en el desempeño académico de alumnos / as de tercer ciclo del centro escolar Santiago Orellana Zelaya de la ciudad de Concepción Batres. Comunicar. 2013;65:1/150.
7. Flores Cueto JJ, Morán Corzo JJ, Rodriguez Vila JJ. Las Redes Sociales. 2009. 2009;1-15.

8. Burgueño Fernández P. Clasificación de Redes Sociales [Internet]. 2009. URL disponible en: https://www. pablofb.com/2009/03 / clasificacion-de-redes-sociales /

9. Leiva Aguilera J. Redes sociales. Situación y tendencias en relación a la Información y la Documentación. Vol. 26, Editoral Baratz. 2009.

10. Ríos Hernández I. El lenguaje: herramienta de reconstrucción del pensamiento. Razón y Palabra. 2014;183-202.

11. Bermúdez Jara FD, Cabrera Vergara S del P, Carranza Gamboa KL. La influencia de las redes sociales en los cambios del registro ortográfico de los estudiantes del tercer grado de nivel secundario de la I.E. César VallejoTrujillo. 2016.

12. Brañez Medina RF. La construcción discursiva de las identidades " amixer " $\mathrm{y}$ " no - amixer " en el espacio virtual . Un caso de racismo cultural justificado a través de la ortografía [Internet]. 2012. URL disponible en: http: / / tesis.pucp.edu.pe/ repositorio / bitstream/ handle / 123456789 / 1618 / BRANEZ_MEDINA_ ROBERTO_CONSTRUCCION.pdf?sequence $=1$

13. Martínez R. La reducción morfológica de palabras en la red social Facebook en estudiantes de cuarto año de educación media general del Liceo Bolivariano Pedro Gual, Valencia, Estado Carabobo [Internet]. Vol. 205, Universidad de Carabobo - Venezuela. 2014. URL disponible en: https://www.cambridge.org/core/ product/identifier/S0007125000277040/type / journal_ article

14. Álvarez Ortiz MN. La influencia de la red social Facebook en el lenguaje escrito de los estudiantes del grado octavo del colegio Jaime Garzón de Cúcuta [Internet]. Vol. 1, Universidad Santo Tomás - Colombia. 2017. URL disponible en: https:// repository.usta.edu. co/bitstream / handle/11634 / 10501 / AlvarezNataly 2017.pdf? sequence $=1 \&$ is Allowed $=y$

\section{CORREPONDENCIA:}

claudia-diaz-cj@hotmail.com 